\title{
Inhibition of the transcription factor Sp1 suppresses colon cancer stem cell growth and induces apoptosis in vitro and in nude mouse xenografts
}

\author{
YINGYING ZHAO*, WENJING ZHANG* , ZHENG GUO*, FENG MA, YAO WU, YANG BAI, WEI GONG, \\ YE CHEN, TIANMING CHENG, FACHAO ZHI, YALI ZHANG, JIDE WANG and BO JIANG
}

Guangdong Provincial Key Laboratory of Gastroenterology, Department of Gastroenterology, Nanfang Hospital, Southern Medical University, Guangzhou, Guangdong 510515, P.R. China

Received March 1, 2013; Accepted May 2, 2013

DOI: 10.3892/or.2013.2627

\begin{abstract}
The transcription factor specificity protein 1 (Sp1) plays a role in the development and progression of various types of human cancers, while cancer stem cells (CSCs) are important in cancer cell self-renewal, resistance to chemotherapy and metastatic potential. This study investigated the role of Sp1 in colon CSC growth and apoptosis. Colon CSCs were successfully enriched using special culture medium and identified by typical CSC gene expression. In a quiescent state, these CSCs formed spheres with slow proliferation; overexpressed Sp1, CD44, CD166 and CD133 proteins; upregulated mesenchymal markers; and a downregulated epithelial marker were noted. In $e x$ vivo experiments, the $\mathrm{Sp} 1$ protein was expressed in $74.8 \%$ of colon cancer tissues, whereas it was expressed only in $42.2 \%$ of the distant normal colon mucosae. Furthermore, inhibition of SP1 expression using Sp1 siRNA or mithramycin A (MIT) led to marked suppression of CSC growth and induced apoptosis. In addition, the percentage of $\mathrm{CD} 44^{+} / \mathrm{CD} 166^{+}$cells was significantly downregulated both in vivo and in vitro following Sp1 inhibition. In conclusion, Sp1 suppression attenuated the characteristics of colon CSCs. Thus, Sp1 inhibition may be potentially useful for the future development of a novel therapeutic strategy to control colon cancer.
\end{abstract}

Correspondence to: Dr Bo Jiang or Dr Jide Wang, Guangdong Provincial Key Laboratory of Gastroenterology, Department of Gastroenterology, Nanfang Hospital, Southern Medical University, Guangzhou, Guangdong 510515, P.R. China

E-mail: drjiang@163.com

E-mail: jidewang@gmail.com

*Contributed equally

Abbreviations: Sp1, specificity protein 1; CSCs, cancer stem cells

Key words: colon cancer, Sp1, CSCs, CD44, CD166

\section{Introduction}

Colorectal cancer is one of the most common cancers in the world with over 1.2 million new cancer cases and over 600,000 deaths annually. The risk factors for colorectal cancer include smoking tobacco; being physically inactive, overweight, or obese; and consuming red and processed meat or excessive alcohol. These factors cause gene mutations and altered gene expression, which promote uncontrolled cell growth and invasion. Increasing evidence indicates that cancer stem cells (CSCs) are responsible for cancer formation, recurrence and drug resistance. Colon CSCs consist of a group of colorectal epithelial cells with the ability to self-renew, which drive tumorigenesis and differentiation, generating the heterogeneous mass within a tumor. These CSCs persist in the tumor mass as a distinct population and cause tumor relapse and metastasis; however, most CSCs are in a quiescent state. In other words, they are in a nondividing state, so they are much less sensitive to classical anti-proliferative chemotherapeutic regimens (1-4). To effectively control colorectal cancer in the clinic, there needs to be a better understanding of colorectal CSCs and their biological behavior, such as identification of growth regulation of CSCs in a tumor mass or cell population and their functions in maintaining stemness and tumorigenicity in colorectal cancer.

To date, a consistent biomarker to precisely identify CSCs has not been identified; although the molecules CD133, CD44, CD166 and EpCAM have been proposed as CSC markers in various types of human cancers $(5,6)$. For example, CD44 has been linked to certain breast and prostate cancers for their CSC properties and has been reported to play a key role in tumor progression and poor prognosis (7). Similarly, CD166 is expressed in aggressive melanoma and breast, colorectal and bladder cancers (8-13). CD166 is also highly expressed within the endogenous intestinal stem cell niche and has been applied as a marker of pluripotent mesenchymal stem cells $(14,15)$.

In addition, specificity protein 1 (Sp1), a human transcription factor involved in gene expression in the early development of an organism, plays an important role in colorectal cancer development and progression. Sp1 contains a zinc finger protein motif that binds to the consensus sequence 5 '-(G/T) GGGCGG(G/A)(G/A)(C/T)-3'. Expression of Sp1 protein 
has been shown to be elevated in different types of tumors, including colorectal cancers and is associated with patient prognosis $(16,17)$. Functionally, Sp1 protein regulates the expression of various genes that are important in tumorigenesis, such as genes related to cell proliferation, differentiation, apoptosis, drug resistance and metastasis $(18,19)$. In this study, we first enriched colon CSCs from colorectal cancer cell lines and tissues and then determined whether Spl protein can regulate the growth and gene expression of these CSCs in order to effectively control colorectal CSCs for the future treatment of colorectal cancer in the clinic.

\section{Materials and methods}

Antibodies and reagents. Rabbit antibodies against Ki-67, Snail, vimentin, c-kit and glyceraldehyde-3-phosphate dehydrogenase (GAPDH) were obtained from Abcam (Cambridge, UK). PE-conjugated mouse anti-human CD166 and IgG1K and FITC-conjugated mouse anti-human CD44 and IgG2b1k were purchased from BD Pharmingen (San Diego, CA, USA). Goat anti-rabbit IgG-FITC, mouse anti-human Sp1, and rabbit anti-human E-cadherin antibodies were purchased from Santa Cruz Biotechnology, Inc. (Santa Cruz, CA, USA). Pyronin Y and the Sp1 inhibitor MIT were products of Sigma (St. Louis, MO, USA).

Cell lines and culture. Colon cancer SW480, HCT116, DLD1, HT29, HCT15, LoVo and SW1116 cell lines were maintained in the laboratory as previously described (19). To enrich colon CSCs, SCM was used for cell culture. It was prepared from Dulbecco's modified Eagle's medium (DMEM), i.e., addition of Nutrient Mixture F12 (DMEM/F12; Gibco-BRL, Rockville, MD, USA) with N-2 Plus Medium Supplement (Invitrogen Life Technologies, Carlsbad, CA, USA), $10 \mathrm{ng} / \mathrm{ml}$ recombinant human fibroblast growth factor-basic (FGF-2), $20 \mathrm{ng} / \mathrm{ml}$ recombinant human epidermal growth factor (EGF) (both from Imgenex Corp. San Diego, CA, USA), $100 \mu \mathrm{g} / \mathrm{ml}$ streptomycin, and $100 \mathrm{U} / \mathrm{ml}$ penicillin, without the addition of serum. The CSCs were cultured in ultra-low attachment dishes or plates (Corning Inc., Lowell, MA, USA) that do not allow adherence to a substratum. The cell culture was maintained in a humidified incubator at $37^{\circ} \mathrm{C}$ with an atmosphere of $95 \%$ air and $5 \%$ carbon dioxide.

Purification of the stem cell fraction. The mononuclear cells were isolated from colorectal cancer cell lines by density gradient centrifugation through Ficoll-Paque ${ }^{\mathrm{TM}}$ PLUS (endotoxin tested, sterile; GE Healthcare). In brief, colorectal cancer SW480 or HCT116 cells were cultured with SCM in dishes coated with an ultra-low attachment surface for 1 week and refed with SCM every day. Next, the tumor cells were carefully loaded into Ficoll media and centrifuged for $30 \mathrm{~min}$ at $1080 \mathrm{rpm}$ with 0 deceleration, and the interphase layer of cells was then collected and washed thoroughly with phosphatebuffered saline (PBS). These enriched colorectal CSCs were further cultured with SCM for the following experiments.

Immunofluorescence staining. Colorectal cancer cells and the enriched colorectal CSCs were collected from the monolayer culture using trypsin, scattered onto glass cover slips using a
Shandon Cytospin 4 (Thermo Electron Corporation, Waltham, MA, USA), and then fixed with $4 \%$ paraformaldehyde. Next, the cells were incubated with the Ki-67 antibody (1:100) for $2 \mathrm{~h}$ followed by the FITC-conjugated secondary antibody (1:100) for $1 \mathrm{~h}$ in the dark. The cell nuclei were counterstained with $1 \mu \mathrm{g} / \mathrm{ml}$ Hoechst 33258. After mounting, images were obtained under a Zeiss Axioscop fluorescence microscope. The proliferation index was expressed as the percentage of CSCs to that of colorectal cancer cells.

Reverse transcription-polymerase chain reaction (RT-PCR) and quantitative RT-PCR ( $q R T-P C R)$. Total RNA was isolated using TRIzol reagent (Gibco-BRL, Grand Island, NY, USA), and complementary DNA (cDNA) was synthesized by a ThermoScript ${ }^{\mathrm{TM}}$ reverse-transcription PCR system (Invitrogen Life Technologies) according to the manufacturer's instructions. PCR was performed using a $25-\mu 1$ system with Hotstart DNA polymerase (Qiagen, Hilden, Germany) for 36 cycles of $95^{\circ} \mathrm{C}$ denaturation for $30 \mathrm{~min}$ for the first cycle and then at $94^{\circ} \mathrm{C}$ for $30 \mathrm{sec}, 53^{\circ} \mathrm{C}$ annealing for $30 \mathrm{sec}$, and $72^{\circ} \mathrm{C}$ elongation for $60 \mathrm{sec}$ plus $72^{\circ} \mathrm{C}$ for $10 \mathrm{~min}$ at the last cycle. The PCR products were separated on a $1 \%$ agarose gel.

qPCR was performed using the Option real-time PCR system (Bio-Rad, Hercules, CA, USA) with Power SYBR-Green PCR Master Mix. Reactions were carried out in duplicate in a $15-\mu 1$ reaction volume. The qPCR conditions were $5 \mathrm{~min}$ at $95^{\circ} \mathrm{C}$, followed by 50 cycles of $95^{\circ} \mathrm{C}$ for $15 \mathrm{sec}$, $56^{\circ} \mathrm{C}$ for $30 \mathrm{sec}$, and $72^{\circ} \mathrm{C}$ for $40 \mathrm{sec}$. A final extension at $72^{\circ} \mathrm{C}$ for $5 \mathrm{~min}$ was included before a temperature ramp from 72 to $95^{\circ} \mathrm{C}$ at $0.1^{\circ} \mathrm{C} / \mathrm{sec}$. Gene expression was normalized to GAPDH (housekeeping gene) and the cycle threshold values were calculated using the $2^{-\Delta \Delta \mathrm{Ct}}$ method. The primer sequences used in this study are listed in Table I.

Spl siRNA construction and transfection. The sequences of Sp1 siRNA and a control siRNA were as follows: 5'-AAAGC GCUUCAUGAGGAGUGA-3' and 5'-UUCUCCGAACGU GUCACGUTT-3', respectively. For these siRNA transfections into cells, colorectal CSCs were grown to 30-50\% confluence and then transfected with Sp1 siRNA or the control siRNA using Lipofectamine 2000 according to the manufacturer's instructions. The effect of gene knockdown was evaluated by qPCR.

Protein extraction and western blotting. Total cellular protein was extracted using RIPA buffer and then quantified using a BCA kit. Next, lysates containing $50 \mu \mathrm{l}$ of protein in each sample were analyzed by SDS-PAGE, and proteins were transferred to a polyvinylidene difluoride membrane (Millipore, Bedford, MA, USA). Residual protein sites were incubated with different primary antibodies followed by the appropriate horseradish peroxidase-conjugated secondary antibody for protein visualization with ECL reagents (Millipore, Billerica, MA, USA).

Flow cytometry. To assess changes in cell cycle distribution in colorectal cancer cells and CSCs, the cells were grown and subjected to flow cytometric analysis. In brief, the cells were first fixed in $70 \%$ ethanol and incubated with PBS containing $2 \mu \mathrm{g} / \mathrm{ml}$ Hoechst 33258 for $15 \mathrm{~min}$. Pyronin $\mathrm{Y}$ was then 
Table I. Primer sequences for PCR or qPCR.

\begin{tabular}{|c|c|c|}
\hline Gene name & Sequence $5^{\prime}-3^{\prime}$ (forward and reverse) & Length (bp) \\
\hline CD133 & $\begin{array}{l}\text { CAAATGTGGTGGAGAAATGC } \\
\text { GTGATTTGCCACAAAACCAT }\end{array}$ & 132 \\
\hline CD44 & $\begin{array}{l}\text { AGACATCTACCCCAGCAACC } \\
\text { GGTGATCCAGGGACTGTCTT }\end{array}$ & 142 \\
\hline CD166 & $\begin{array}{l}\text { TCTGCTCTTCTGCСTCTTGA } \\
\text { CGGGCTTTTCATATTTCCAT }\end{array}$ & 155 \\
\hline Sp1 & $\begin{array}{l}\text { TGGATGAGGCACTTCTGTCA } \\
\text { GAAGGTGCCTGCGTCAGTAG }\end{array}$ & 134 \\
\hline Snail & $\begin{array}{l}\text { AGACGAGGACAGTGGGAAAG } \\
\text { AGATCCTTGGCCTCAGAGAG }\end{array}$ & 168 \\
\hline E-cadherin & $\begin{array}{l}\text { GTCAGGTGCCTGAGAACGAG } \\
\text { GCCATCGTTGTTCACTGGAT }\end{array}$ & 158 \\
\hline Vimentin & $\begin{array}{l}\text { GAACTTTGCCGTTGAAGCTG } \\
\text { TCTCAATGTCAAGGGCCATC }\end{array}$ & 143 \\
\hline c-kit & $\begin{array}{l}\text { CCGAAGGAGGCACTTACACA } \\
\text { GAATCCTGCTGCCACACATT }\end{array}$ & 146 \\
\hline GAPDH & $\begin{array}{l}\text { GTCAACGGATTTGGTCGTATTG } \\
\text { CTCCTGGAAGATGGTGATGGG }\end{array}$ & 216 \\
\hline
\end{tabular}

PCR, polymerase chain reaction; qPCR, quantitative polymerase chain reaction; Sp1, specificity protein 1; GAPDH, glyceraldehyde-3-phosphate dehydrogenase.

added at $4 \mu \mathrm{g} / \mathrm{ml}$. Fluorescence of 10,000 cells/sample was measured after $20 \mathrm{~min}$. Apoptotic cells were detected using the FITC-Annexin V Apoptosis Detection Kit I (BD Biosciences, San Diego, CA, USA). Cells were harvested and resuspended in $500 \mu \mathrm{l}$ of $1 \mathrm{X}$ binding buffer. A total of $10 \mu \mathrm{l}$ of FITC-Annexin V and $2 \mu \mathrm{g} / \mathrm{ml}$ propidium iodide (PI) were added to the cell mixture and incubated for $15 \mathrm{~min}$ in the dark prior to analysis. To observe the expression of stem cell surface markers, cells were stained with anti-CD44-FITC, anti-CD166-PE, or the isotype-matched control IgG for $45 \mathrm{~min}$. After washing, cells were fixed with $0.5 \%$ paraformaldehyde prior to FACS analysis.

Tissue specimens. A total of 45 pairs of colorectal cancer and distant normal tissue specimens were collected at the Department of Gastroenterology, Nanfang Hospital (Guangzhou, China). The paraffin blocks were prepared for immunohistochemistry. This study was approved by the Medical Ethics Committee of Nanfang Hospital, and all patients provided written informed consent.

Tissue specimens and immunohistochemistry. For immunohistochemistry experiments, paraffin-embedded tissue sections were prepared and then deparaffinized and rehydrated. The endogenous peroxidase activity was blocked by incubation with hydrogen peroxide, and antigen retrieval was then performed by incubation in a pepsin solution at $37^{\circ} \mathrm{C}$. The sections were then incubated with an anti-Sp1 antibody $(1: 200)$ at $4{ }^{\circ} \mathrm{C}$ overnight, followed by incubation with the biotin-linked anti-mouse IgG (Dako, Copenhagen, Denmark) and then with the ABC complex. The staining sections were then reviewed and scored according to our previous published criteria (17). In particular, the cells with $<10 \%$ staining were scored as negative staining (-), while $10-49 \%$ staining as,$+ 50-74 \%$ as ++ , and $75-100 \%$ as +++ .

Cell viability MTT assay and colony formation assay. To assess the effects of Sp1 knockdown in colorectal CSCs, we transiently transfected Sp1 siRNA into the enriched CSCs. Alternatively, we also treated them with 500 nM MIT, a Sp1 inhibitor (16). Next, viable cells were counted up to 3 days. For the colony formation assay, we plated the cells $\left(1 \times 10^{4}\right)$ in 35-mm dishes with SCM containing $0.3 \%$ top agar and $0.6 \%$ bottom agar and then grew them for 14 days with observation every 3 days. At the end of the experiment, the cell colonies were stained with $0.5 \%$ violet blue and counted. Colonies containing $>50$ cells were counted.

Animal experiment. The animal experiment was approved by the Committee on the Ethics of Animal Experiments of Southern Medical University. Briefly, 5-6-week-old BALB/c nude mice were divided into 4 groups with 3 mice/group. SW480 or HCT116 cells $\left(2 \times 10^{6}\right)$ with $>90 \%$ viability were subcutaneously injected into the right flank of each mouse. After the tumors became palpable, $25 \mathrm{mg} / \mathrm{kg}$ of MIT or PBS as a control $(n=3)$ was given intraperitoneally every 3 days for 3 weeks. After the last treatment, tumors were removed and digested into a single-cell suspension for detection of CD44 and CD166 expression using FACS analysis. 

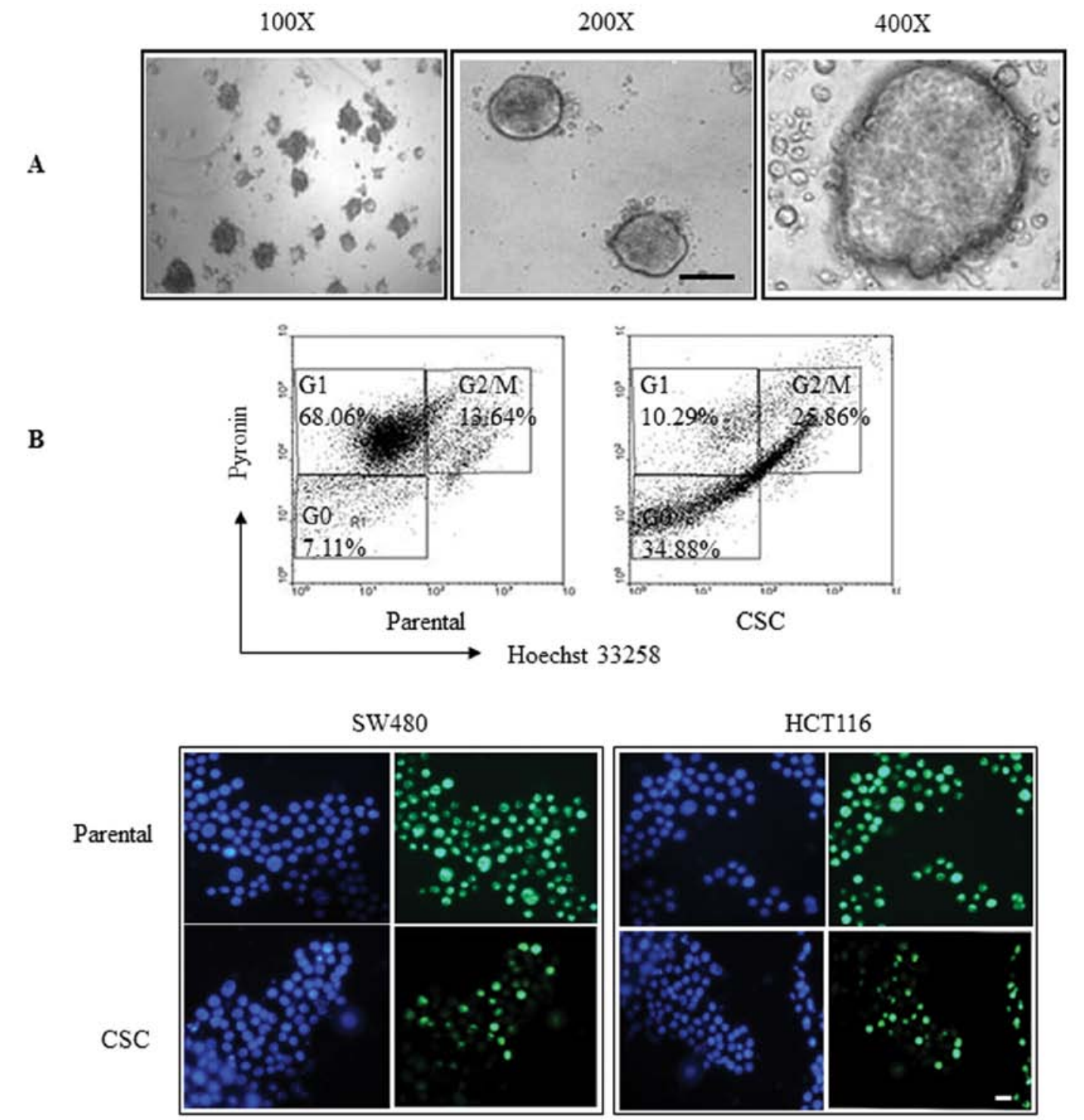

C
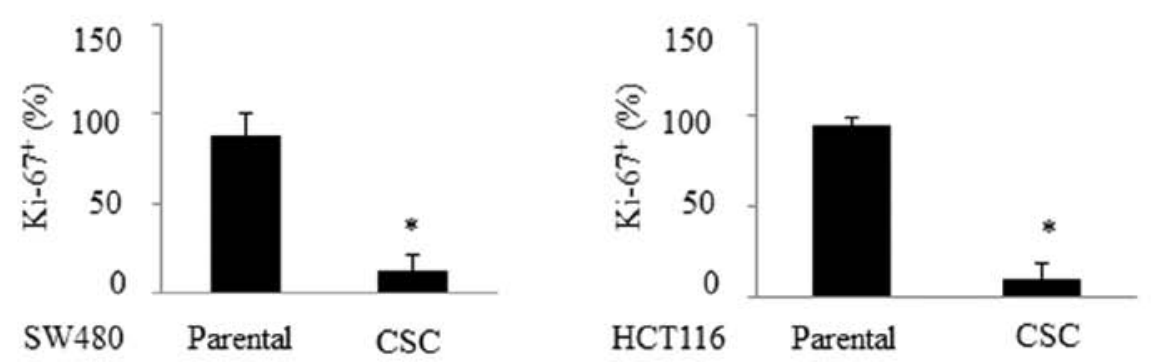

Figure 1. Isolation and identification of colon cancer stem cells (CSCs). (A) Stem cell spheres isolated from SW480 cells. The bar represents $100 \mu \mathrm{m}$. (B) Fluorescence-activated cell sorting (FACS) analysis. CSCs were fixed and stained with $2 \mu \mathrm{g} / \mathrm{ml} \mathrm{Hoechst} 33258$ and $4 \mu \mathrm{g} / \mathrm{ml}$ Pyronin Y for FACS analysis. The scale bar represents $20 \mu \mathrm{m}$. (C) Immunohistochemistry. Ki-67 expression was assessed using an anti-Ki-67 antibody and a FITC-conjugated secondary antibody. Hoechst 33258 was used to stain the cell nuclei. ${ }^{*} \mathrm{P}<0.05$, compared to the parental cells. All of these experiments were repeated 3 times.

Statistical analysis. All data are presented as means \pm standard deviation (SD), and the differences between groups were evaluated by the two-sided independent samples t-test. Statistical significance was set at $\mathrm{P} \leq 0.05$.

\section{Results}

Enrichment and identification of a colorectal CSC population from colorectal cancer cells. In this study, we performed special cell culture of colorectal cancer cell lines with stem cell medium (SCM) for Ficoll isolation. We then collected the middle interface cells from the Ficoll isolation and observed the enriched CSCs under a microscope. As shown in Fig. 1A, these cells were large and round in unattached floating spheroid colonies. Fluorescence-activated cell sorting (FACS) analysis showed that the percentage of quiescent cells in the stem spheres was greater compared with that in the parental cells (34.88 vs. 7.11\%; Fig. 1B). Moreover, we immunofluorescently 
A

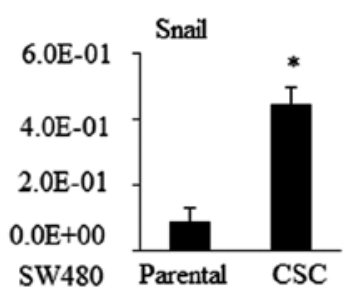

B

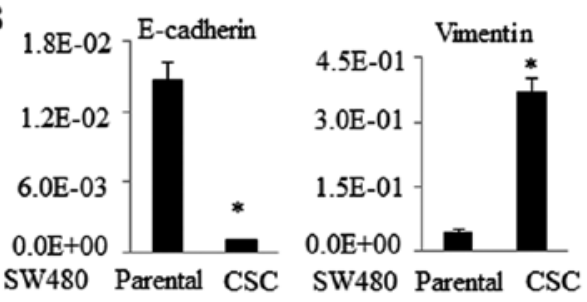

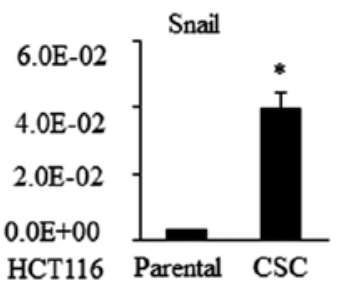

C
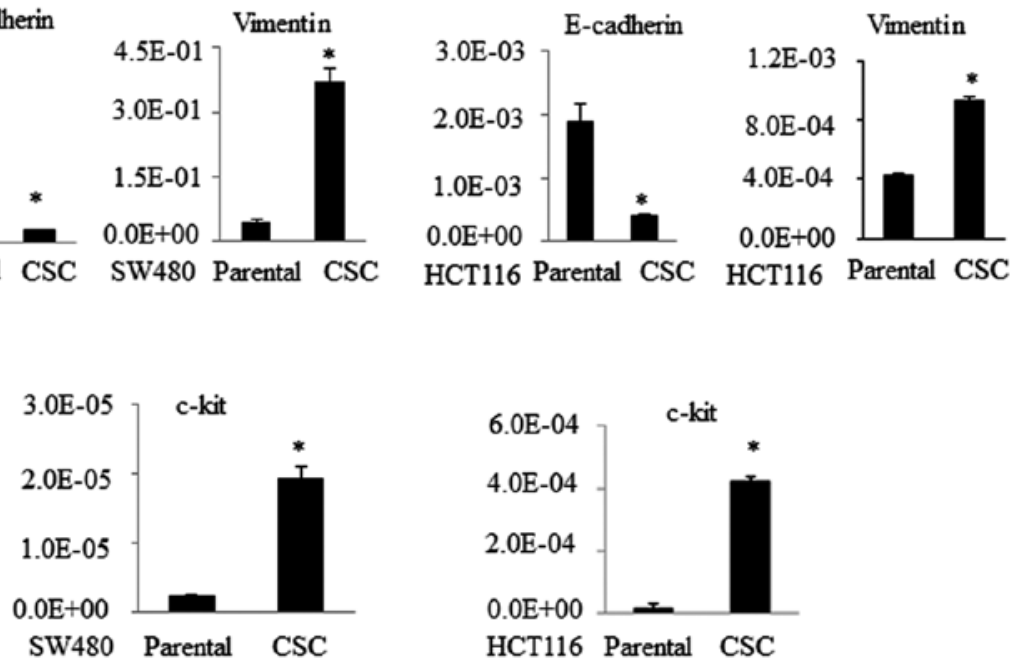

D

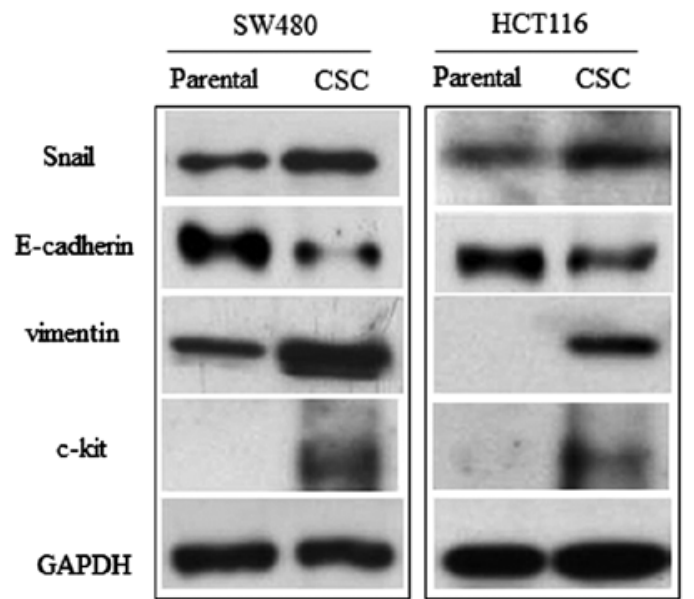

Figure 2. Expression of epithelial-mesenchymal transformation (EMT) markers in colon cancer stem cells (CSCs). Expression of (A) Snail, (B) E-cadherin and vimentin and (C) c-kit was analyzed by qPCR and (D) western blotting in CSCs and their parental cells with GAPDH used as an internal control.

stained them for Ki-67 (a cell-division marker) and found that the percentage of $\mathrm{Ki}$-67-positive cells was $12.29 \%$ in the SW480 CSC spheres compared to $87.06 \%$ in the parental SW480 cells $(\mathrm{P}<0.05)$, while the percentage of Ki-67-positive cells was $9.9 \%$ in the HCT116 CSC spheres compared to $94 \%$ in the parental HCT116 cells $(\mathrm{P}<0.05$; Fig. 1C). These findings suggest that these CSCs grew much more slowly than the parental cells.

In addition, we detected the expression of additional genes in these CSCs. For example, expression of pluripotency genes and acquisition of mesenchymal markers have been regarded as stem cell phenotypes (20). We assessed expression of Snail [an epithelial mesenchymal transformation (EMT)-activating transcription factor], E-cadherin, and vimentin (2 EMT regulatory proteins) in these CSCs. We found that expression levels of Snail and vimentin mRNA were significantly greater in CSCs compared to those in parental SW480 or HCT116 cells. In contrast, E-cadherin expression was less in CSCs (Fig. 2A and B). Similarly, CD117 (c-kit) is a stem cell factor receptor involved in cell signaling transduction in several cell types (21). CD117 expression in SW480 and HCT116 cells was very low or even undetectable, but it was significantly greater in CSCs (Fig. 2C and D). These data suggest that these cells are CSCs.

Differential expression of cell surface markers in CSCs and parental cells. Next, we detected the expression of 3 commonly accepted stem cell surface markers (CD133, CD44 and CD166) 
A
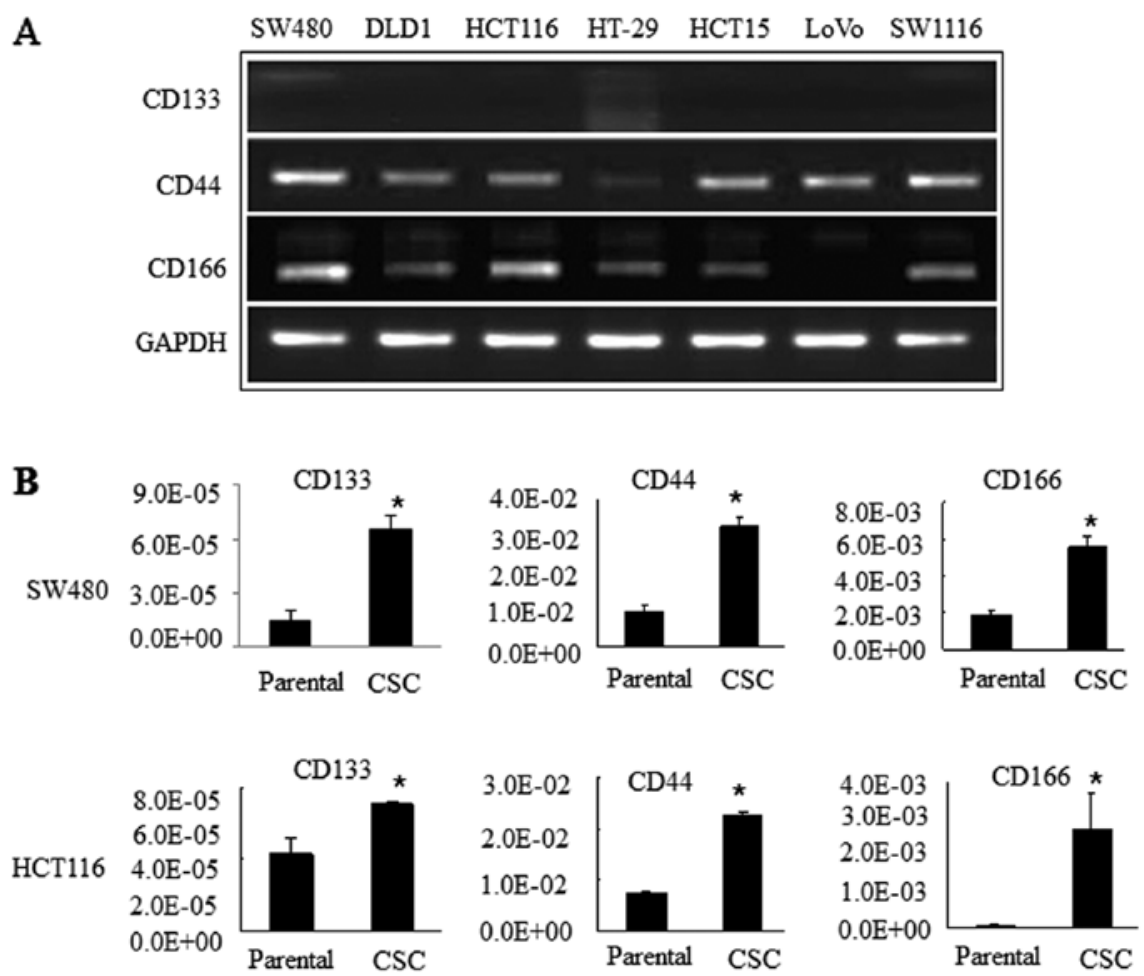

C

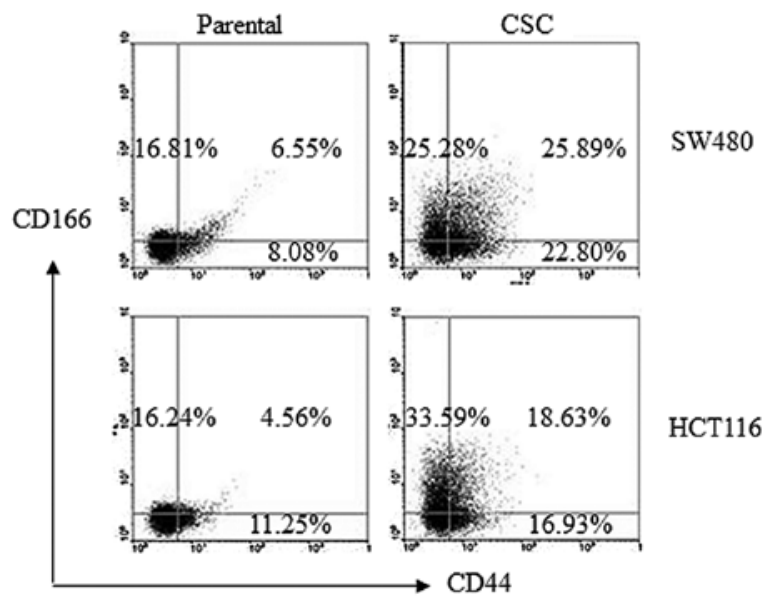

Figure 3. Expression of stem cell surface markers in colon cancer cell lines and cancer stem cells (CSCs). (A) Expression of CD133, CD44 and CD166 proteins was analyzed using RT-PCR in 7 cell lines. GAPDH was used as an internal control. Expression of CD44 and CD166 was evaluated in SW480 or HCT116 spheres and parental cells by (B) qPCR and (C) FACS analysis. These experiments were repeated 2-3 times with similar findings.

in 7 different colon cancer cell lines. Our data showed that CD44 was expressed in all 7 colorectal cancer cell lines, i.e., SW480, DLD1, HCT116, HT29, HCT15, LoVo and SW1116, while CD166 was expressed in all cell lines except LoVo. In contrast, CD133 was expressed at very low levels or was even undetectable in these 7 cell lines (Fig. 3A). We then assessed their expression levels in the CSCs derived from SW480 and HCT116 cells and found that all 3 of these surface markers were significantly upregulated in CSCs compared to the parental cells. In particular, CD44 and CD166 were expressed at a level of $10^{-2}-10^{-3} /$ cell, while CD133 was $10^{-5} /$ cell in CSCs from both SW480 and HCT116 cells (Fig. 3B). Furthermore, the percentage of $\mathrm{CD}_{4} 4^{+} / \mathrm{CD}_{166}{ }^{+}$cells was $25.89 \%$ in SW480 CSC spheres compared to $6.55 \%$ in SW480 cells. Similarly, there were $18.63 \%$ of $\mathrm{CD}^{2} 4^{+} / \mathrm{CD}_{166}{ }^{+} \mathrm{HCT} 116 \mathrm{CSC}$ spheres compared to $4.56 \%$ in HCT116 cells (Fig. 3C). These data indicate that CD44 and CD166 may be useful to define CSCs from colon cancer.

Overexpression of Spl protein in colon cancer tissues. We further confirmed the overexpression of Sp1 in 45 pairs of human distant normal and cancerous colon tissue specimens. Fig. 4A illustrates the typical staining of representative tumor and normal mucosa specimens. The quantitative data on all 45 patients revealed that normal colon tissues had $42.2 \%$ Sp1-positive and only 5.9\% strong Sp1-positive cells, whereas colon cancer tissues had $74.8 \% \mathrm{Sp1}$-positive and $30.3 \%$ strong Sp1-positive cells (10.4, +; 34.1, ++; 30.3\%, +++; Fig. 4B). Similar results were found with CSCs and their parental cells (Fig. 4C and D). These data demonstrated that Sp1 was 
$\mathbf{A}$
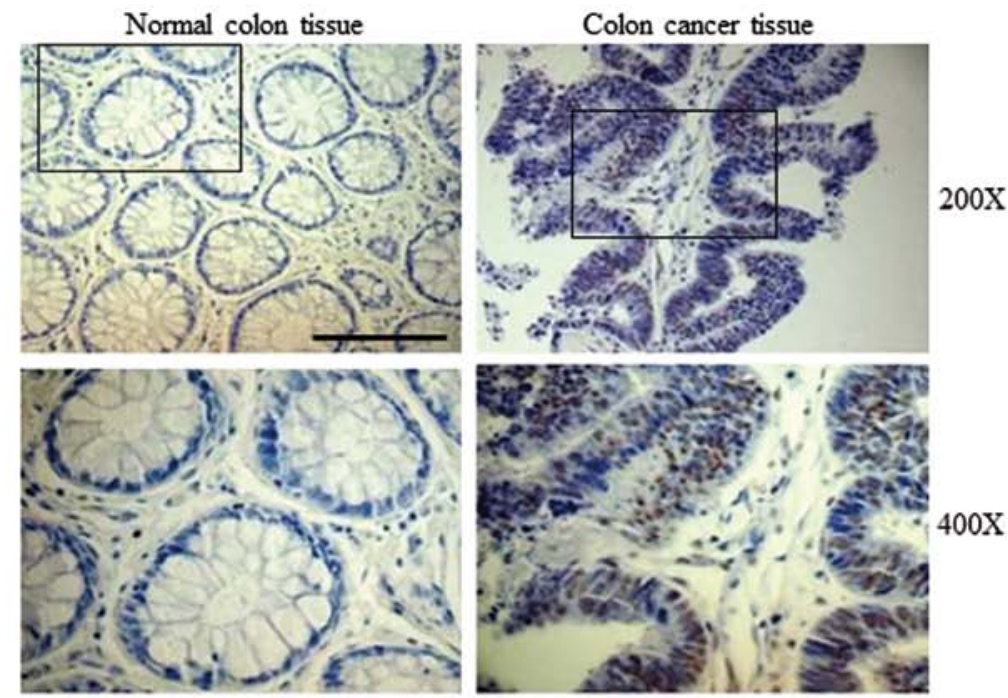

B
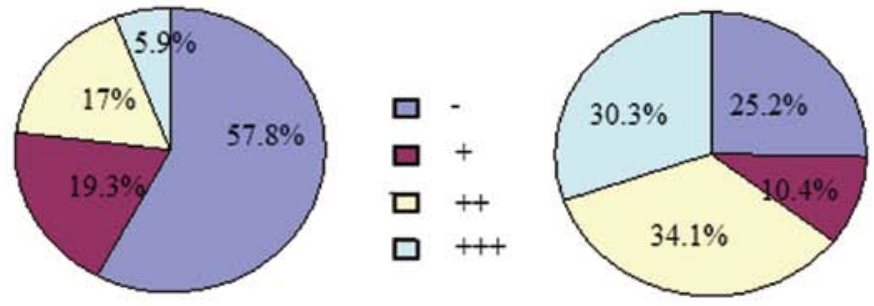

C

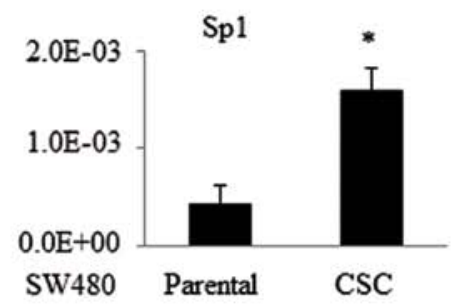

$\mathrm{Sp} 1$

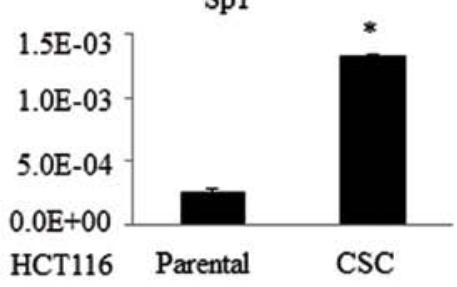

D

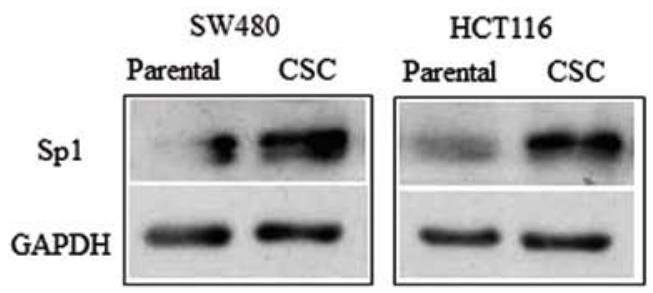

Figure 4. Sp1 expression in colon cancer tissues and cancer stem cells (CSCs). (A) Immunohistochemistry staining and (B) quantitative analysis of Sp1 in 45 pairs of distant normal and colon cancer tissues. The scale bar represents $20 \mu \mathrm{m}$. Sp1 expression was detected in SW480 and HCT116 CSC spheres and the parental cells using (C) qPCR and (D) western blotting with GAPDH as an internal control.

expressed at a greater level in human colon cancer specimens than in adjacent normal colon tissues, suggesting that $\mathrm{Sp} 1$ protein may be useful to identify a colon CSC population in cells and tissues.

Effects of Sp1 inhibition on suppression of CSC growth and induction of CSC apoptosis. Next, we determined the effects of Sp1 knockdown on the regulation of CSC growth and apoptosis by transient Sp1 siRNA transfection or treatment with the Sp1 inhibitor mithramycin A (MIT). We found that
Sp1 siRNA transfection into CSCs prevented growth of these CSCs (Fig. 5A). The soft agar assay showed that Sp1 inhibition sharply reduced colony formation from 100 to $9.3 \%$ in the siRNA group and $4.65 \%$ in MIT-treated cells (Fig. 5B). In parallel, CSC apoptosis was increased after Sp1 inhibition, i.e., Sp1 siRNA transfection induced CSC apoptosis by $43.94 \%$ and MIT treatment induced CSC apoptosis by 50.7\% (Fig. 5C).

Effects of Spl inhibition on the regulation of CD44 and CD166 expression in vitro and in nude mice. Next, we 
$\mathbf{A}$

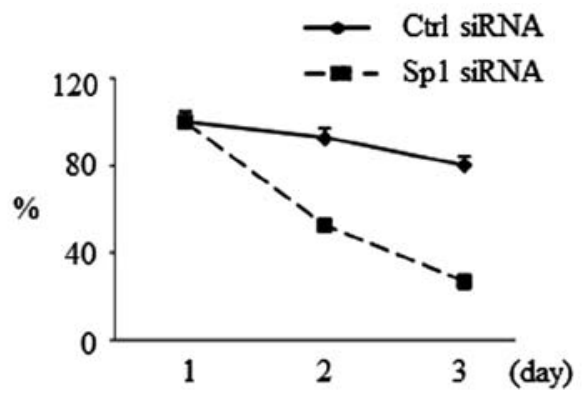

B

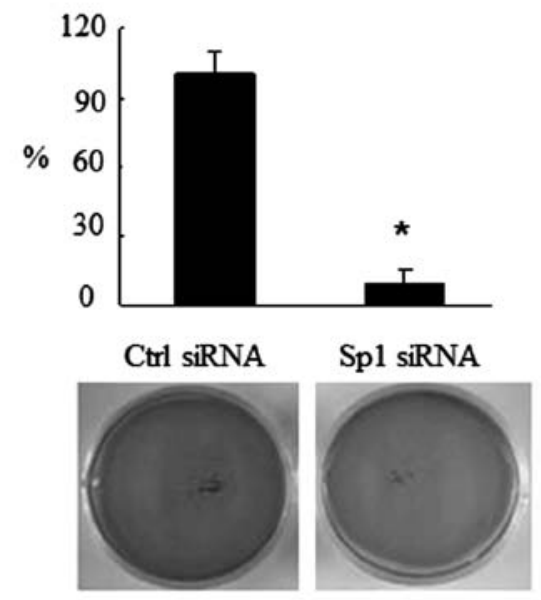

\section{C}

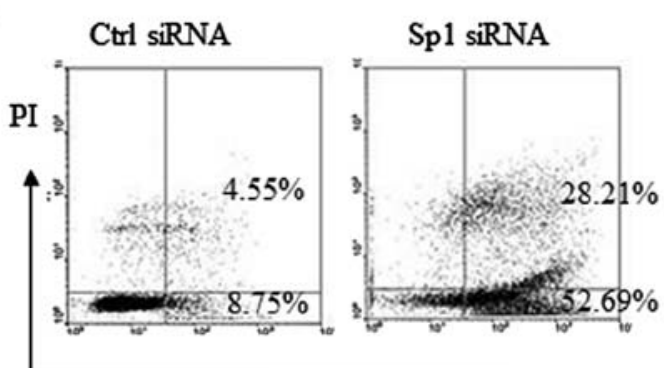

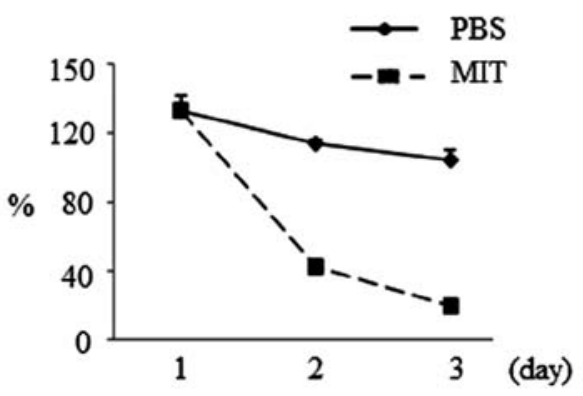
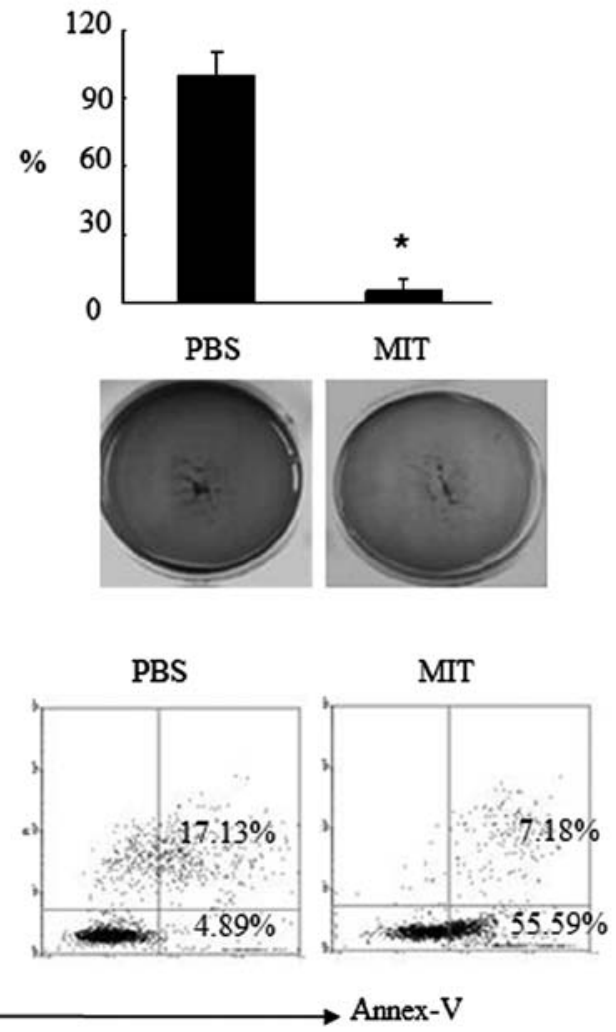

Figure 5. Effect of Sp1 inhibition on the regulation of colon cancer stem cell (CSC) growth, colony formation and apoptosis. (A) CSCs were transfected with control or Sp1 siRNA or treated with or without $500 \mathrm{nM}$ MIT for the indicated time points. The average number of viable cells in 5 fields/group was counted at a magnification, $\mathrm{x} 200 .{ }^{*} \mathrm{P}<0.05$, compared to the control siRNA or PBS group. (B) Colony formation assay. The duplicate CSCs (1x $\left.10^{4}\right)$ were seeded into 35 -mm dishes in $0.3 \%$ top agar and $0.6 \%$ bottom agar and grown for 14 days. At the end of the experiments, cell colonies ( $\geq 50$ cells) were counted under a microscope. The results were expressed as percentages relative to control cells ( ${ }^{*} \mathrm{P}<0.05$, between control siRNA and Sp1 siRNA, or between PBS and MIT). (C) Flow cytometric apoptosis assay. Control or Sp1 siRNA or MIT-treated CSCs were cultured in SCM for 48 h. Apoptosis was detected by FACS analysis after double staining with PI and Annexin-V-FITC. All of these experiments were repeated 2-3 times.

explored the effect of Sp1 inhibition on suppressing the expression of CD44 and CD166 in vitro and in nude mice. As shown in Fig. 6A, CD44 expression was inhibited by $76.89 \%$, and CD166 expression was reduced by $37.05 \%$ after Sp1 knockdown by Sp1 siRNA in SW480 CSC spheres. Consistently, MIT treatment also inhibited CD44 and CD166 expression in a dose-dependent manner (Fig. 6B). FACS data showed that Sp1 siRNA transfection resulted in $6.77 \% \mathrm{CD}^{4} 4^{+} / \mathrm{CD} 166^{+}$ cells compared to $32.05 \%$ in the control siRNA group, while MIT treatment decreased $\mathrm{CD} 44^{+} / \mathrm{CD} 166^{+}$expression from 20.08 to $0.93 \%$ (Fig. 6C).

Furthermore, we also performed nude mouse experiments by injecting SW480 and HCT116 cells into mice. After the tumors became palpable, we treated these mice with $25 \mathrm{mg} / \mathrm{kg}$ of MIT or PBS as a control. At the end of the experiment, tumor tissue was resected and subjected to FACS analysis. The data showed that the percentage of $\mathrm{CD} 44^{+} / \mathrm{CD} 166^{+}$cells in the tumors of MIT-treated mice was $2.14 \%$ compared to $18.02 \%$ in the tumors of the SW480 cell-injected mice. Consistently, there were 2.9 and $23.6 \%$ of $\mathrm{CD} 44^{+} / \mathrm{CD} 166^{+}$cells, respectively, with or without MIT treatment in the tumors of the HCT116 cell-injected mice (Fig. 6D). Of note, MIT treatment had a greater effect on CD44 inhibition than on CD166 inhibition.

\section{Discussion}

In this study, we successfully enriched CSCs from colorectal cancer SW480 or HCT116 cell lines by using a special serumfree cultivation method. These enriched CSCs displayed typical stem cell properties; for example, cell quiescence with 
$\mathbf{A}$

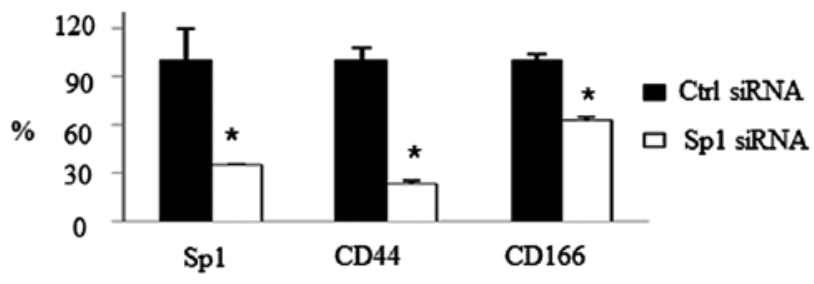

B
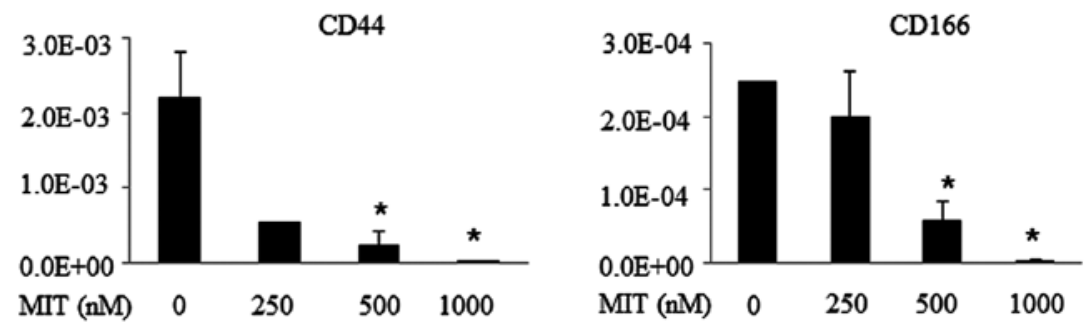

C

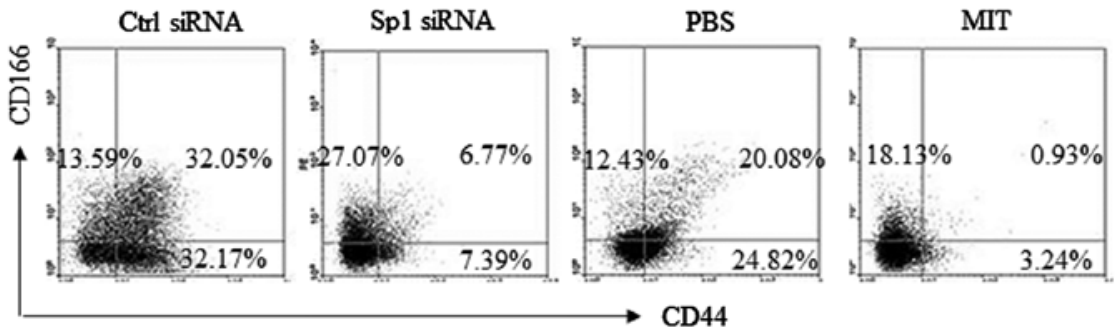

D

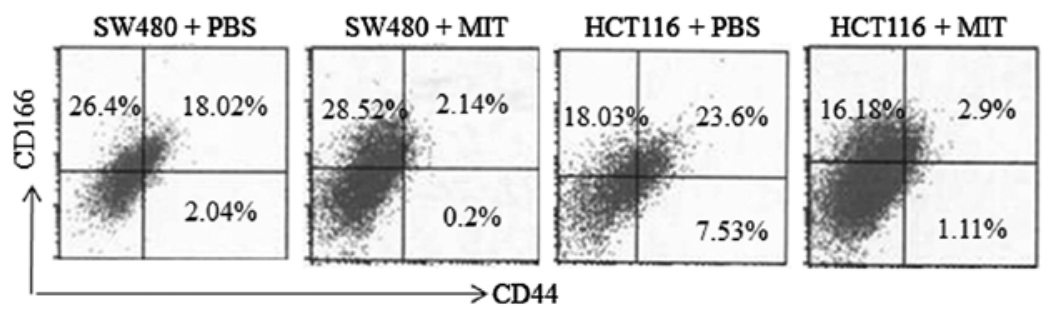

Figure 6. Effect of Sp1 inhibition on the regulation of CD44 and CD166 expression in vitro and in nude mouse xenografts. (A) qRT-PCR. SW480 cancer stem cells (CSCs) were transfected with Sp1 siRNA or control siRNA for $48 \mathrm{~h}$. qRT-PCR was performed to detect the expression of Sp1, CD44 and CD166. (B) The duplicate CSCs were subjected to the indicated concentrations of MIT for $48 \mathrm{~h}$ followed by qPCR to detect CD44 and CD166 expression. (C) SW480 CSCs were transfected with Sp1 siRNA or control siRNA, or they were subjected to MIT treatment for $48 \mathrm{~h}$. Then, FACS analysis was used to determine CD44 and CD166 expression levels. (D) Nude mouse xenograft experiments. SW480 and HCT116 cells were inoculated subcutaneously into 5-6-week-old BALB/c nude mice $(n=3)$. After the tumor became palpable, MIT or PBS as a control was intraperitoneally injected every 3 days for 3 weeks. At the end of the experiments, tumor xenografts were digested into single-cell suspensions and subjected to FACS analysis after double staining with anti-CD44-FITC and anti-CD166-PE antibodies.

slow growth and expression of stemness markers (e.g., CD44, CD166 and c-kit), mesenchymal markers (vimentin and Snail), and Sp1. Furthermore, Sp1 inhibition suppressed the growth of these CSCs but promoted apoptosis. These data suggest that targeting Sp1 protein could be useful for the development of a novel therapeutic strategy to control colon cancer.

To date, serum-free cell culture and cancer spheroid cell formation are the main methods with which to enrich or isolate CSCs (22). However, most studies focus on isolation from clinical specimens with mechanical dissociation and collagenase digestion (23-25). In the present study, we enriched CSCs from 2 different colon cancer cell lines, which excluded any mesenchymal cells or stromal tissues. Furthermore, our current data showed that expression of CD133, CD44 and CD166 proteins was cell line-specific. Although the hypothesis that a tumor may originate from a single CSC has been raised for many years, not all colon cancer cell lines expressed equally high levels of stemness markers. This result may be due to the long-term self-renewal potential of CSCs and their ability to generate heterogeneous progeny.

Recent studies have demonstrated close associations among tumor EMT, cancer metastasis and CSCs (26-28). Vimentin is an important mesenchymal marker, and Snail is a significant transcription factor of EMT. In our study, the expression of both vimentin and Snail was upregulated in these CSCs. These results are consistent with previous studies that showed a direct link between EMT and a gain of epithelial stem cell properties $(26,27)$. Moreover, c-kit protein plays a critical role 
in the growth and differentiation of various types of cells, including hematopoietic stem cells. A previous study found that in salivary adenoid cystic carcinoma, c-kit overexpression was associated with tumor cell invasion and metastasis (29). Most importantly, Sp1 is not only required for EMT, but it also is able to bind to the c-kit promoter, thereby inhibiting c-kit gene transcription $(30,31)$. Thus, the present study linked Sp1 and c-kit together, which may suggest that Sp1 confers not only EMT but also CSC activity.

Furthermore, CD133 was initially regarded as a marker of tumor-initiating cells and has been used to isolate CSCs from fresh lung cancers. However, its role as a marker of colon CSCs has been subsequently challenged. In the present study, almost all of the 7 cell lines studied showed low levels of CD133 expression. Similar results were found by Chen et al (32) (only $0.7 \%$ CD133 expression in H1299 cells) and Leung et al (20) (no CD133 expression in tumor cells). These studies indicate that CD133 may not be a useful marker for CSCs in certain types of cancer.

In the present study, CD44 and CD166 were differentially expressed in various colon cancer cell lines. These results may indicate that expression of stem cell surface markers are both tissue-specific and cell line-specific. Similar discrepant observations have also been shown in ovarian and liver cancer cells. The inconsistent expression may be due to different potency states and compositional or functional characteristics of the CSC or progenitor populations (20). Interestingly, CD44 expression was significantly decreased after Sp1 inhibition using siRNA knockdown or MIT treatment, whereas such an inhibitory effect on CD166 expression was not obvious. Nevertheless, the underlying molecular mechanisms need to be further elucidated.

In addition, the expression level of Sp1 protein was much greater in colon cancer tissues than in normal colon tissues. Similar results were found in CSCs, i.e., Sp1 expression was significantly greater in colon CSCs than in parental colon cancer cells. These data suggest that $\mathrm{Sp} 1$ expression is a potential marker associated with colon disease progression. Sp1 siRNA and MIT treatment suppressed CSC sphere growth and induced apoptosis in vitro; Sp1 suppression also attenuated CD44 and CD166 expressions in vivo, suggesting that Sp1 expression also has a close relationship with CSCs. Sp1 knockdown may not only attenuate the malignant phenotype of colon cancer, but it also decreases the survival of colon CSCs.

In conclusion, this study demonstrated that $\mathrm{Sp} 1$ was overexpressed in colon CSCs. Inhibition of Sp1 suppressed the traits of CSCs and promoted cell apoptosis. Hence, the self-renewal ability, drug resistance, and metastasis potential of colon CSCs may be partially due to preferentially high expression of Sp1 protein. Our findings link the transcription factor $\mathrm{Sp} 1$ to colon CSCs for the first time and indicate that Sp1 suppression may be a potential therapeutic strategy for colon cancer.

\section{Acknowledgements}

This study was supported by NSFC grants (30973404, 81172057 and 81272761), President Foundation of Nanfang Hospital (2012B009), high-level topic matching funds of Nanfang Hospital (2010036, G201227) and Twelfth Five-year- plan (2011BAZ03191) from the National Technology Support Program.

\section{References}

1. Vermeulen L, Sprick MR, Kemper K, Stassi G and Medema JP: Cancer stem cells - old concepts, new insights. Cell Death Differ 15: 947-958, 2008.

2. Gupta PB, Chaffer CL and Weinberg RA: Cancer stem cells: mirage or reality? Nat Med 15: 1010-1012, 2009.

3. O'Brien CA, Kreso A and Dick JE: Cancer stem cells in solid tumors: an overview. Semin Radiat Oncol 19: 71-77, 2009.

4. Lonardo E, Hermann PC, Mueller MT, et al: Nodal/Activin signaling drives self-renewal and tumorigenicity of pancreatic cancer stem cells and provides a target for combined drug therapy. Cell Stem Cell 9: 433-446, 2011.

5. Anderson EC, Hessman C, Levin TG, Monroe MM and Wong MH: The role of colorectal cancer stem cells in metastatic disease and therapeutic response. Cancers 3: 319-339, 2011.

6. Horst D, Kriegl L, Engel J, Kirchner T and Jung A: Prognostic significance of the cancer stem cell markers CD133, CD44, and CD166 in colorectal cancer. Cancer Invest 27: 844-850, 2009.

7. Thorne RF, Legg JW and Isacke CM: The role of the CD44 transmembrane and cytoplasmic domains in co-ordinating adhesive and signalling events. J Cell Sci 117: 373-380, 2004.

8. Ofori-Acquah SF and King JA: Activated leukocyte cell adhesion molecule: a new paradox in cancer. Transl Res 151: 122-128, 2008.

9. van Kempen LC, van den Oord JJ, van Muijen GN, Weidle UH, Bloemers HP and Swart GW: Activated leukocyte cell adhesion molecule/CD166, a marker of tumor progression in primary malignant melanoma of the skin. Am J Pathol 156: 769-774, 2000.

10. King JA, Ofori-Acquah SF, Stevens T, Al-Mehdi AB, Fodstad O and Jiang WG: Activated leukocyte cell adhesion molecule in breast cancer: prognostic indicator. Breast Cancer Res 6: R478-R487, 2004.

11. Burkhardt M, Mayordomo E, Winzer KJ, et al: Cytoplasmic overexpression of ALCAM is prognostic of disease progression in breast cancer. J Clin Pathol 59: 403-409, 2006.

12. Weichert W, Knösel T, Bellach J, Dietel M and Kristiansen G: ALCAM/CD166 is overexpressed in colorectal carcinoma and correlates with shortened patient survival. J Clin Pathol 57: 1160-1164, 2004.

13. Tachezy M, Zander H, Gebauer F, et al: Activated leukocyte cell adhesion molecule (CD166) - its prognostic power for colorectal cancer patients. J Surg Res 177: e15-e20, 2012.

14. Levin TG, Powell AE, Davies PS, et al: Characterization of the intestinal cancer stem cell marker CD166 in the human and mouse gastrointestinal tract. Gastroenterology 139: 2072-2082, 2010.

15. van Kilsdonk JW, Wilting RH, Bergers M, et al: Attenuation of melanoma invasion by a secreted variant of activated leukocyte cell adhesion molecule. Cancer Res 68: 3671-3679, 2008.

16. Campbell VW, Davin D, Thomas S, et al: The G-C specific DNA binding drug, mithramycin, selectively inhibits transcription of the C-MYC and C-HA-RAS genes in regenerating liver. Am J Med Sci 307: 167-172, 1994.

17. Guo Z, Zhang W, Xia G, et al: Sp1 upregulates the four and half lim 2 (FHL2) expression in gastrointestinal cancers through transcription regulation. Mol Carcinog 49: 826-836, 2010.

18. Tian H, Qian GW, Li W, et al: A critical role of Sp1 transcription factor in regulating the human Ki-67 gene expression. Tumour Biol 32: 273-283, 2011.

19. Jungert K, Buck A, von Wichert G, et al: $\mathrm{Spl}$ is required for transforming growth factor- $\beta$-induced mesenchymal transition and migration in pancreatic cancer cells. Cancer Res 67: 1563-1570, 2007.

20. Leung EL, Fiscus RR, Tung JW, et al: Non-small cell lung cancer cells expressing CD44 are enriched for stem cell-like properties. PLoS One 5: e14062, 2010.

21. Sperling C, Schwartz S, Buchner T, Thiel E and Ludwig WD: Expression of the stem cell factor receptor C-KIT (CD117) in acute leukemias. Haematologica 82: 617-621, 1997.

22. Lee J, Kotliarova S, Kotliarov Y, et al: Tumor stem cells derived from glioblastomas cultured in bFGF and EGF more closely mirror the phenotype and genotype of primary tumors than do serum-cultured cell lines. Cancer Cell 9: 391-403, 2006. 
23. Liu S, Dontu G, Mantle ID, et al: Hedgehog signaling and Bmi-1 regulate self-renewal of normal and malignant human mammary stem cells. Cancer Res 66: 6063-6071, 2006.

24. Dey D, Saxena M, Paranjape AN, et al: Phenotypic and functional characterization of human mammary stem/progenitor cells in long term culture. PLoS One 4: e5329, 2009.

25. Tomuleasa C, Soritau O, Rus-Ciuca D, et al: Isolation and characterization of hepatic cancer cells with stem-like properties from hepatocellular carcinoma. J Gastrointestin Liver Dis 19: 61-67, 2010.

26. Sigurdsson V, Hilmarsdottir B, Sigmundsdottir H, et al: Endothelial induced EMT in breast epithelial cells with stem cell properties. PLoS One 6: e23833, 2011.

27. Tellez CS, Juri DE, Do K, et al: EMT and stem cell-like properties associated with miR-205 and miR-200 epigenetic silencing are early manifestations during carcinogen-induced transformation of human lung epithelial cells. Cancer Res 71: 3087-3097, 2011.
28. Singh A and Settleman J: EMT, cancer stem cells and drug resistance: an emerging axis of evil in the war on cancer. Oncogene 29: 4741-4751, 2010.

29. Tang Y, Liang X, Zheng M, et al: Expression of c-kit and Slug correlates with invasion and metastasis of salivary adenoid cystic carcinoma. Oral Oncol 46: 311-316, 2010.

30. Maeda K, Nishiyama C, Ogawa $\mathrm{H}$ and Okumura K: GATA2 and $\mathrm{Sp} 1$ positively regulate the c-kit promoter in mast cells. J Immunol 185: 4252-4260, 2010.

31. Lecuyer E, Herblot S, Saint-Denis M, et al: The SCL complex regulates c-kit expression in hematopoietic cells through functional interaction with Sp1. Blood 100: 2430-2440, 2002.

32. Chen YC, Hsu HS, Chen YW, et al: Oct-4 expression maintained cancer stem-like properties in lung cancer-derived CD133-positive cells. PLoS One 3: e2637, 2008. 